Mots. Les langages du politique

Les livres de journalistes politiques

\title{
Isabelle Léglise et Nathalie Garric éd. Discours
} d'experts et d'expertise

2012, Berne, Peter Lang, 226 pages

Claire Oger

\section{(QpenEdition}

\section{Journals}

Édition électronique

URL : https://journals.openedition.org/mots/21652

DOI : $10.4000 /$ mots. 21652

ISSN : 1960-6001

Éditeur

ENS Éditions

Édition imprimée

Date de publication : 19 mai 2014

Pagination : 119-122

ISBN : 978-2-84788-542-2

ISSN : 0243-6450

\section{Référence électronique}

Claire Oger, «Isabelle Léglise et Nathalie Garric éd. Discours d'experts et d'expertise », Mots. Les

langages du politique [En ligne], 104 | 2014, mis en ligne le 19 mai 2016, consulté le 23 avril 2022. URL : http://journals.openedition.org/mots/21652 ; DOl : https://doi.org/10.4000/mots.21652 


\section{Compte rendu de lecture}

\section{Discours d'experts et d'expertise}

Isabelle Léglise et Nathalie Garric éd.

2012, Berne, Peter Lang, 226 pages

Intitulé Discours d'experts et d'expertise et publié aux éditions Peter Lang en 2012, l'ouvrage dirigé par Isabelle Léglise et Nathalie Garric est venu combler une lacune : les recherches menées en sociologie ou en science politique ont produit une imposante bibliographie pour rendre compte de la place et du rôle des experts dans les sociétés contemporaines, bibliographie diversifiée qui tout à la fois éclaire et reflète les débats auxquels ont donné lieu leurs transformations (place de l'expertise dans l'élaboration des politiques publiques, relation des experts aux institutions, montée en puissance d'une « expertise profane »...). Or les travaux menés dans le cadre théorique de l'analyse du discours ou dans des perspectives proches, s'ils existaient à l'état dispersé, n'avaient pas encore été rassemblés dans un ouvrage susceptible de fournir un aperçu des principales directions de travail actuellement explorées sur le sujet.

L'ouvrage se concentre sur les discours produits par des experts «patentés», ou «en situation d'expertise », entendue ici comme un dispositif communicationnel spécifique articulant trois instances : une autorité dotée d'une certaine légitimité, une instance «citoyenne » et une instance experte (p.7). En ce sens, la production des discours d'experts est envisagée en relation avec une situation d'énonciation bien définie et ce positionnement constitue un mérite de l'ouvrage, qui articule par là la question des relations entre pouvoir et savoir (ou savoir-faire) à celle de la crédibilité construite en discours. Il contribue pourtant aussi à dessiner deux limites du projet éditorial, que l'on évoquera rapidement avant de souligner le grand intérêt des contributions rassemblées.

Un certain nombre de lecteurs regretteront peut-être que les liens avec les recherches sociologiques et les débats qui les traversent ne soient pas suffisamment explicités dans l'introduction (ou dans une conclusion générale) qui, sans renoncer à un point de vue discursif, aurait pu opérer sur ce point des rapprochements plus précis et développer des références qui restent un peu allusives dans les premières pages. L'apport spécifique de l'analyse du discours serait d'ailleurs ressorti plus nettement d'une mise en perspective plus détaillée.

Autre effet restrictif des choix initiaux : en centrant la réflexion sur l'expertise comme dispositif communicationnel, l'ouvrage dégage un certain nombre de caractéristiques linguistiques et discursives observées dans les corpus étudiés, mais ne croise guère d'autres travaux, menés pourtant dans le même 
champ de recherche, notamment les propositions faites par Roser Cusso et Corinne Gobin dans le numéro 88 de la revue Mots. Les langages du politique (2008) consacré aux « discours experts » : en étendant la désignation aux discours institutionnels, c'est moins la fonction ou le rôle de l'expert qui y sont visés que l'analyse de ces discours comme productions idéologiques, auxquelles contribuent construction de l'évidentialité, étayage par la doxa aussi bien que légitimation de la «rationalité» politique. Une situation des contributions de l'ouvrage, qui manient elles aussi de telles catégories, par rapport à ces propositions, aurait été bienvenue.

Ces remarques doivent être lues comme des regrets plutôt que comme des réserves car la discussion sur ces points aurait sûrement renforcé la portée de l'ouvrage et elles n'affectent pas la cohérence du propos, construit autour de trois volets : les médias - et les relations entre journalisme et expertise -, la justice - et l'articulation entre jugement et rapports d'experts -, l'expertise "scientifique » enfin - et la relation $\mathrm{au}(\mathrm{x})$ savoir(s) dans les débats « citoyens ».

Aurélie Tavernier s'intéresse dans le premier chapitre à la manière dont les rhétoriques du journalisme d'information mobilisent les discours d'experts et singulièrement la parole rapportée du sociologue. Satisfaisant à la double prétention à l'objectivité, d'une part, au traitement de la complexité d'autre part, la mobilisation de l'autorité du sociologue intervient de manière privilégiée autour de thématiques spécifiques (famille et éducation, violences urbaines, conjugalité, tourisme estival) et sous la forme différenciée de la citation, de l'interview ou de la tribune. Elle est ainsi «convoquée pour opérer une requalification des faits journalistiques en catégories d'analyse scientifique» (p.38), où la «description objectivée» par le chiffre alimente une rhétorique positiviste (p.40). Elle se fait aussi normative en passant du diagnostic à la prescription, mais apparaît en définitive davantage comme un moyen de renforcer la crédibilité du journaliste que comme une manifestation de l'autorité de l'expert lui-même.

Pierre Lejeune s'intéresse quant à lui aux relations entre Notes de conjoncture de l'Insee et articles de la rubrique économique du Monde et y observe un mouvement de convergence symétrique : alors que Le Monde tend à abandonner la « rhétorique d'expertise critique » qui le caractérisait (p.49), relayant de plus en plus le discours de l'institution, l'Insee, pour sa part, évolue vers un discours plus «vendeur» que strictement scientifique. Cette fine étude diachronique, qui compare la période 1987-1994 aux années 2007-2008, permet d'observer les transformations de l'ethos des journalistes aussi bien que celui des experts et d'examiner en particulier la part accordée à l'évaluation et à l'axiologie dans les deux discours, et le déplacement de l'un à l'autre de la dimension argumentative du propos.

Le volet relatif à la justice s'ouvre sur une contribution de Caroline Protais, consacrée à une controverse d'experts opposant deux collèges de psychiatres : la question posée est celle de l'imputation de responsabilité par les juges en 
fonction du diagnostic psychiatrique posé par les experts, l'«abolition du discernement» étant rarement prononcée. L'analyse montre comment la controverse étudiée se soutient de «conceptions cliniques divergentes, en particulier concernant la définition donnée à la réalité délirante » (p. 90) mais aussi de positions différentes sur le lien entre maladie mentale et comportement criminel, et même sur la possibilité d'une « objectivité médico-légale » (p. 95). L'empreinte du courant antipsychiatrique et de l'approche critique de l'exclusion des malades mentaux se lit dans l'une de ces postures, tandis que l'autre se satisfait davantage d'une orthodoxie plus technicienne. La rareté du non-lieu psychiatrique, sans pouvoir être rapportée à cette seule ligne de clivage, comme le rappelle la conclusion, reçoit ici un éclairage qui souligne les enjeux sociaux des divergences théoriques et éthiques qui parcourent un tel corps d'experts.

En s'attachant au contraire aux caractères partagés de l'expertise judiciaire, Laurence Dumoulin met en évidence un autre fonctionnement (évoqué par ailleurs dans des travaux très différents qui ne sont pas cités ici par l'auteure, comme ceux de Robert Castel ou de Nicolas Dodier). Observation, constatation, explication dominent en apparence dans le rapport d'expertise supposé alimenter la réflexion du juge sans se prononcer sur les catégories juridiques. Audelà pourtant de cette distinction de principe entre fait et droit, il apparaît que la dimension argumentative du propos fait affleurer le jugement moral, ainsi qu'une évaluation de la normalité des comportements (par exemple dans le domaine éducatif). Normalisation tendancielle et intériorisation (ou anticipation) des catégories de la qualification juridique contribuent, dans cette perspective, à faire du rapport d'expertise une préfiguration de jugement.

Dans le troisième volet de l'ouvrage, intitulé «Expertise et savoir », Frédéric Lebaron fait tout d'abord remarquer comment la discipline économique s'est imposée comme un acteur majeur de l'évaluation des politiques publiques en même temps que s'imposait le «modèle anglo-saxon» qui avait établi sa position dominante aux États-Unis puis au Royaume-Uni. Instances privées de représentation professionnelle et institutions publiques de référence contribuent au succès d'une pratique centrée sur le «chiffrage », la production d'indicateurs, le benchmarking... C'est dans un tel contexte qu'il convient d'aborder le débat sur le «modèle social français » qui oppose schématiquement un pôle libéral et un pôle social-démocrate; il aboutit pour ce dernier à la tentative de promouvoir un «modèle scandinave» et une «flexisécurité» qui sont mobilisés dans des argumentations ambiguës et aboutissent, en définitive, à la valorisation paradoxale d'un modèle américain qui s'impose implicitement comme seule référence. La valorisation indirecte de ce modèle fait ainsi écho à l'américanisation de la discipline économique dans le champ académique.

C'est un paradoxe d'une autre nature qu'éclaire la contribution suivante, dans laquelle Anna-Célia Disdier et Valélia Muni Toke se penchent sur l'exemple de la gestion du risque alimentaire. Mobilisé dans un contexte de controverse, 
le discours d'expert s'éloigne constitutivement des catégories scientifiques (certitude, probabilité) pour glisser vers un «jugement de plausiblité», mouvement que vient renforcer l'appréhension qualitative (plutôt que quantitative) du risque par les citoyens. La préférence donnée en France à une «gouvernance technique » exercée par des institutions qui émettent des «avis» et des recommandations explique la faible médiatisation de certains sujets relatifs au risque alimentaire et contraste avec l'implication plus grande du consommateur citoyen observée auxÉtats-Unis. Bien plus, contre-expertise et coconstruction des discours d'experts apparaissent comme un horizon commun à l'ensemble des nouveaux modèles de gestion du risque.

Le dernier chapitre de l'ouvrage, rédigé par Marianne Doury et Marie-Cécile Lorenzo-Basson, s'intéresse à la conférence de citoyens sur les OGM qui a eu lieu en France en 1999. Il s'agit ici d'examiner le fonctionnement détaillé d'un dispositif de démocratie participative et d'y interroger la légitimité attribuée aux participants «experts» ou «profanes» à travers les notions de cadrage et de rôle telles que définies par Goffman (p.181). L'examen de l'organisation séquentielle de la conférence (formation puis conférence proprement dite, sous forme de tables rondes, puis délibération, puis annonce publique de l'avis) n'est pas un simple préalable à l'analyse puisque cette séquentialité est mentionnée dans le fil même des débats, notamment par les experts. La distribution prévue des rôles contraint fortement le déroulement des débats et place les experts sous le contrôle du président de séance qui distille approbation ou réserves. Mais ce dispositif ne détermine pas entièrement le déroulement des échanges qui laisse émerger, dans les tables rondes, des prises de position critiques ou polémiques entre citoyens et experts ou entre les experts eux-mêmes, tendant à rétablir des formes de communication symétrique. Les enchaînements questions-réponses au contraire rétablissent l'asymétrie des compétences entre citoyens et experts. Ceux-ci sont également séparés par des conceptions différentes de l'expertise, les experts valorisant la contradiction tandis que les citoyens se défient des divergences d'analyse. Cette contribution constitue un apport majeur à deux titres: tout d'abord parce qu'elle s'efforce de dégager les rôles et la légitimité des uns et des autres à partir d'une étude détaillée des échanges observés dans le cadre d'un dispositif précis (dimension qui souffre d'un effacement relatif dans les travaux sur la démocratie participative, parfois plus attentifs aux rôles prévus qu'aux interactions réalisées). Mais surtout, elle permet d'examiner ce dispositif à l'échelle de ses différentes phases, différenciant fortement le fonctionnement des tables rondes et celui des enchaînements questions-réponses.

En cela et à travers la diversité des cas étudiés, l'ouvrage met en garde contre les typologies généralisantes et les positionnements normatifs qui parcourent parfois la littérature scientifique relative à l'expertise.

Claire Oger

Université Paris 13, Labsic (EA 1803) 